\title{
Estudo anatômico da veia braquial comum como via de drenagem colateral do membro superior
}

\section{Anatomic study of the common brachial vein as a collateral drainage channel of the upper limb}

Carlos Adriano Silva dos Santos', Luiz Francisco Poli de Figueiredo², Luiz Carlos Buarque de Gusmão³, Aldemar Araújo Castro $^{4}$, Guilherme Benjamin Brandão Pitta ${ }^{5}$, Fausto Miranda Jr ${ }^{6}$, Érica Carla Figueirêdo de Souza ${ }^{7}$

\section{Resumo}

Contexto: Traumatismos ou tromboses que possam evoluir com alterações da drenagem venosa do membro superior, dependendo do território interrompido, podem ter como mecanismo compensatório uma via colateral de drenagem sem que haja prejuízo para o retorno venoso desse membro. A veia braquial comum apresenta-se como uma alternativa plausível e pouco conhecida.

Objetivo: Descrever a anatomia da veia braquial comum como via de drenagem colateral no membro superior.

Métodos: Utilizamos 30 cadáveres do sexo masculino, cujos membros superiores estavam articulados ao tronco, não importando a raça, formolizados e mantidos em conservação com solução de formol a 10\%. Utilizamos como critérios de exclusão cadáveres com um dos membros desarticulado ou alterações deformantes em topografia das estruturas estudadas.

Resultados: A veia braquial comum esteve presente em 73\% (22/30) dos cadáveres estudados, sendo que em 18\% (04/22) dos casos drenou para a veia basílica no seguimento proximal do braço e em 82\% (18/22), para a veia axilar.

Conclusão: A veia braquial comum está frequentemente presente e, na maior parte das vezes, desemboca na veia axilar.

Palavras-chave: Veia axilar; cadáver; anatomia; traumatismos do braço; trombose venosa profunda de membros superiores.

\begin{abstract}
Background: Trauma and thrombosis that can result in changes in the venous drainage of the upper limb, depending on the vascular territory interrupted, may have as a compensatory mechanism a collateral drainage channel that prevents damage to the venous return of that limb. The common brachial vein is a plausible and little known collateral channel for this purpose.

Objective: To describe the anatomy of the common brachial vein as a collateral drainage channel of the upper limb.

Methods: We have dissected 30 cadavers of people of different races, whose upper limbs were articulated to the trunk and preserved in a 10\% formaldehyde solution. The exclusion criteria were disarticulated limbs or deformities in the topography of the studied structures.

Results: The common brachial vein was present in $73 \%(22 / 30)$ of the cadavers dissected. The common brachial vein drained into the axillary vein in $82 \%$ (18/22) and into the basilic vein in the proximal segment of the upper limb in $18 \%$ of the cadavers (04/22).

Conclusion: The common brachial vein is frequently present, and, in most cases, it drains into the axillary vein.
\end{abstract}

Keywords: Axillary vein; cadaver; anatomy; wound; upper extremity deep vein thrombosis.

Este trabalho foi apresentado anteriormente no Congresso Brasileiro de Angiologia e Cirurgia Vascular realizado em Maceió (AL), Brasil, no ano de 2009.

${ }^{1}$ Cirurgião Vascular da Unidade de Emergência Dr. Daniel Houly, Arapiraca (AL), Brasil.

2 Professor Titular de Técnica Operatória da Universidade de São Paulo, São Paulo (SP), Brasil.

${ }^{3}$ Doutor em Morfologia; Professor Adjunto do Departamento de Morfologia da Universidade Federal de Alagoas (UFAL), Maceió (AL), Brasil.

${ }^{4}$ Mestre em Cirurgia Vascular; Professor Assistente da Universidade Estadual de Ciências da Saúde de Alagoas (Uncisal), Maceió (AL), Brasil.

${ }^{5}$ Doutor; Professor adjunto, departamento de cirurgia, Universidade Estadual de Ciências da Saúde de Alagoas (Uncisal), Maceió (AL), Brasil.

${ }^{6}$ Professor Adjunto; Livre-docente, departamento de Cirurgia, Universidade Federal de São Paulo (UNIFESP), São Paulo (SP), Brasil.

${ }^{7}$ Acadêmica de Medicina da Universidade Estadual de Ciências da Saúde de Alagoas (Uncisal), Maceió (AL), Brasil.

Submetido em: 04.04.10. Aceito em: 08.12.10

J Vasc Bras. 2011;10(1):40-43. 


\section{Introdução}

Lesões traumáticas graves das veias do membro superior, principalmente na região de transição braço-axila, são responsáveis por um índice elevado de morbidade, além de representar um grande desafio para os cirurgiões ${ }^{1,2}$. Muitas vezes se faz necessária abordagem cirúrgica complexa com maior demanda de tempo e de perda sanguínea, sendo comum nessas lesões a interposição de enxertos, fator de aumento do tempo cirúrgico. Alguns autores têm optado, em situações mais complexas, pela simples ligadura do vaso acometido $^{3-5}$. Salientamos ainda que as veias axilar e braquiais são sede de trombose com potencial risco de embolização ${ }^{6}$, podendo evoluir para síndrome pós-trombótica ${ }^{7}$.

A veia braquial comum (VBC) é formada a partir da união de duas outras veias (veia braquial lateral e veia braquial medial). Origina-se no braço e constitui uma importante via de circulação colateral do membro superior, unindo o braço à axila ${ }^{8-13}$.

A variação de vias colaterais de drenagem venosa que ocorre nesse segmento do membro superior tem importância não só no conhecimento anatômico mas também em procedimentos cirúrgicos nessa região. Por essa razão, foi realizado o presente estudo, que teve como objetivo descrever a anatomia da VBC como via de drenagem colateral do membro superior.

\section{Materiais e métodos}

O estudo foi aprovado pelo Comitê de Ética em Pesquisa da Universidade Estadual de Ciências da Saúde de Alagoas (Uncisal) e Universidade Federal de Alagoas (UFAL).

Consiste em um estudo descritivo de anatomia macroscópica em cadáveres humanos conservados em formaldeído realizado nos laboratórios de anatomia da UFAL e da Uncisal. Tivemos como amostra os membros superiores articulados ao tronco de 30 cadáveres formolizados.

Foram incluídos os cadáveres adultos, do sexo masculino, não importando a raça, formolizados e que apresentavam os membros superiores articulados ao tronco. Excluímos os cadáveres que apresentavam alterações morfológicas nos membros superiores como tumoração, utilização prévia de alguns dos vasos relacionados anatomicamente com o vaso estudado, fratura óssea e lacerações que envolviam as estruturas que compõem o conteúdo vascular e nervoso do braço.

A técnica de dissecação consistiu em colocar o cadáver em decúbito dorsal com o membro superior em abdução (aproximadamente $90^{\circ}$ ), no qual foi efetuada uma incisão longitudinal na face medial do membro, desde a axila até o epicôndilo medial do úmero, associando-se a outras incisões nesse braço e na parede torácica (Figura 1). Após as incisões traçadas, promovemos uma dissecação detalhada por planos das estruturas vasculares e nervosa do braço e axila. Na parede torácica, a musculatura peitoral (peitoral maior e menor) era dissecada e deslocada cranialmente, assim pudemos identificar melhor os vasos e nervos da axila. Para que pudéssemos identificar melhor a VBC, dividimos o braço em três porções (proximal, médio e distal); esses segmentos eram equidistantes entre si, limitados a partir de uma linha traçada entre epicôndilos do úmero (distalmente) e outra linha a partir da borda inferior do músculo redondo maior (proximalmente). $\mathrm{O}$ valor obtido da distância entre esses dois limites era dividido por três e o resultado correspondia ao comprimento dos segmentos (Figura 2).

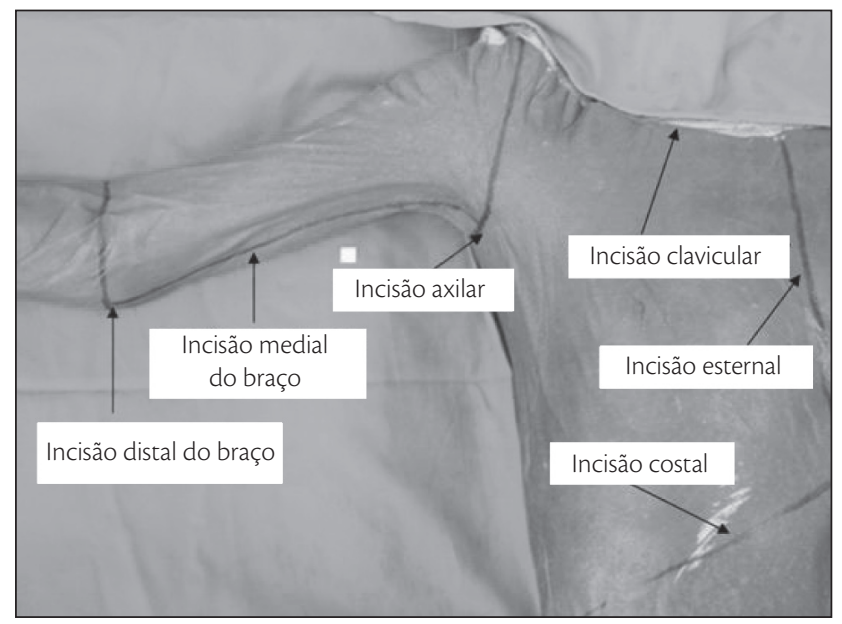

Figura 1 - Cadáver em decúbito dorsal com membro superior em abdução, identificando as linhas de incisões cutâneas

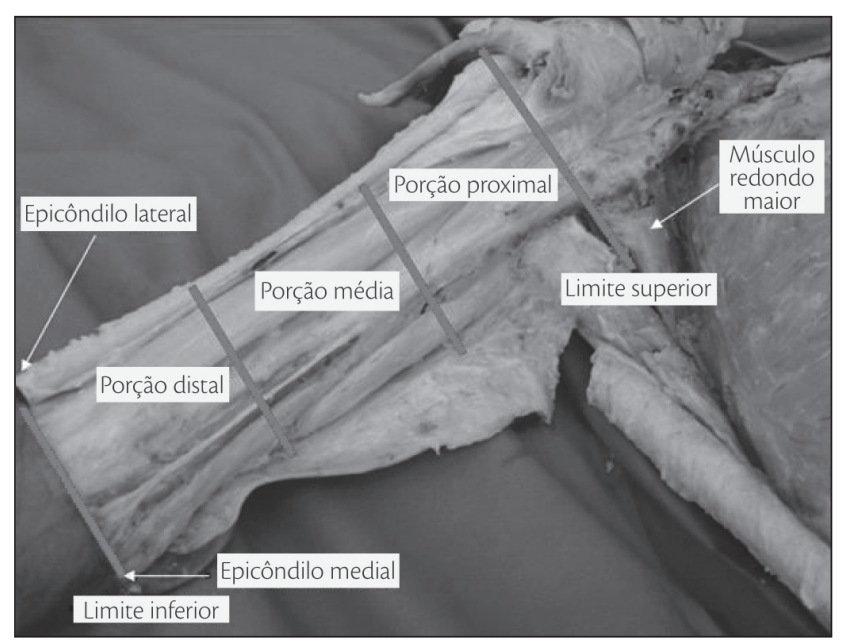

Figura 2 - Identificação dos limites superior e inferior do braço e sua segmentação em porções proximal, média e distal 
Depois de dissecada, procurávamos identificar o nível em que se dava a formação da VBC e sua continuidade ao longo do membro superior.

Para avaliar as variáveis frequência e continuidade da VBC, utilizamos o teste do SINAL. Foi adotado o valor de $\mathrm{p} \leq 5 \%(\alpha \leq 5 \%)$ para rejeitar a hipótese de nulidade.

\section{Resultados}

A VBC esteve presente em 73\% (22/30; IC95\%: 57-89\%; $\mathrm{P} \alpha=0,0081)$ dos cadáveres estudados, sendo que 10 cadáveres $(45 \%)$ apresentavam origem dessa veia no braço esquerdo, 9 (41\%) no braço direito e 3 (14\%) bilateralmente.

Dos 22 cadáveres com VBC, 3 apresentavam essa veia bilateralmente, totalizando 25 veias braquiais comuns em 22 cadáveres. Oitenta e dois por cento (18/22) dos cadáveres apresentaram veias braquiais comuns que ultrapassaram o limite superior do braço. Somente em quatro cadáveres (18\%) essa veia desembocou, ainda no braço, na veia basílica, sendo o terço proximal o de escolha. Foi significante a diferença entre o número de veias que desembocou na basílica do número que desembocou na veia axilar $(\mathrm{P} \alpha=0,0072)$. Excluindo-se as 4 veias braquiais comuns que desembocaram na veia basílica ainda no braço, restaramnos as outras 21 veias que seguiram além do limite superior do braço. Afluindo à veia axilar, a veia braquial comum desembocou no seguimento infrapeitoral em 24\% (5/21); no seguimento peitoral em 43\% (9/21) (Figura 3) e no seguimento suprapeitoral em $33 \%$ (7/21) das veias braquiais comuns. Observamos maior número de VBC desembocando nas porções peitoral e suprapeitoral da veia axilar quando comparado à porção infrapeitoral $(\mathrm{P} \alpha=0,0266)$. A VBC desembocou na veia axilar de 10 membros superiores direito $e$ 11 membros superiores esquerdo (Tabela 1).

\section{Discussão}

O natural da VBC, e de acordo com o encontrado em nosso estudo, é que ela continue além do limite superior do braço. A viabilidade de essa veia continuar como uma importante via de drenagem colateral do membro superior lhe garante um mérito a mais. Na possibilidade ou necessidade de ligadura ou uso demasiado da veia axilar, saber que existe outra via de drenagem torna as situações críticas mais aceitáveis. A maioria das VBC penetrou na axila, poucas ficaram no braço e, mesmo assim, quando neste limitado, a porção proximal do braço foi o local de escolha. Na axila, a VBC desemboca preferencialmente nas porções peitoral e suprapeitoral da veia axilar. Salienta-se o fato de que, quanto mais alto se faz essa desembocadura na veia axilar, mais

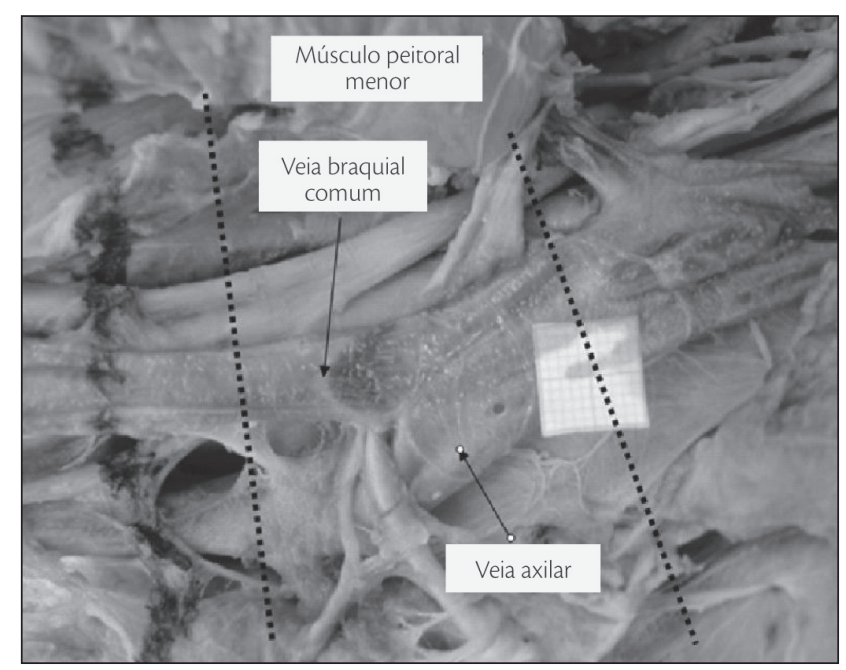

Figura 3 - Desembocadura da veia braquial comum na veia axilar em sua região peitoral.

A área limitada pelas linhas tracejadas corresponde à cobertura do músculo peitoral menor.

Tabela 1 - Desembocadura da veia braquial comum nos membros superiores direito e esquerdo dos cadáveres estudados

\begin{tabular}{|c|c|c|c|c|c|}
\hline \multirow{2}{*}{ Lado } & \multirow{2}{*}{$\begin{array}{c}\text { Veia } \\
\text { basílica }\end{array}$} & \multicolumn{3}{|c|}{ Veia axilar } & \multirow{2}{*}{$\begin{array}{c}\text { Total de } \\
\text { VBC }\end{array}$} \\
\hline & & Infrapeitoral & Peitoral & Suprapeitoral & \\
\hline Direito & 2 & 2 & 4 & 4 & 2 \\
\hline Esquerdo & 2 & 3 & 5 & 3 & 13 \\
\hline Total de VBC & 4 & 5 & 9 & 7 & 25 \\
\hline
\end{tabular}

$P \alpha=0,0072$ para variável desembocadura da veia braquial comum $(V B C)$ na veia basílica em comparação à veia axilar; $P \alpha=0,0266$ para variável desembocadura da $\vee B C$ nas porções peitoral e suprapeitoral em comparação à infrapeitoral da veia axilar.

relevante se torna essa drenagem colateral, pois se aumenta a extensão de veia axilar que pode ser excluída.

É importante citar que Gusmão e Prates ${ }^{11}$ chamam de veia axilar acessória apenas as veias que desembocam no segmento peitoral e suprapeitoral da veia axilar. Dessa forma, eles enfatizam que a drenagem colateral é mais eficaz.

Gerard $^{8}$ citou a existência de um canal coletor que drena as veias circunflexas ou que se continua a partir da veia braquial lateral, classificando esse canal como uma pequena veia axilar que, posteriormente, desembocará na verdadeira veia axilar. Salvi ${ }^{7}$ informa a possibilidade de a veia braquial acessória continuar como veia axilar, sugerindo uma possível veia axilar acessória, citando ainda que esta possa desembocar na veia subclávia. Hollinshead ${ }^{10}$ relatou que a VBC pode densembocar na veia axilar, não citando o nível em que essa desembocadura pode se dar. Gusmão $0^{11}$ descreveu que a veia axilar acessória foi formada pela veia braquial lateral em $55,9 \%$, pela veia braquial comum em $33,4 \%$ e pela veia braquial profunda em apenas $11,8 \%$ dos casos. Santos ${ }^{12}$ 
referiu que $100 \%$ das veias braquiais comuns continuaram como veia axilar acessória e ressaltou a importância dessa veia como via de escoamento colateral.

Considerando que as vias de drenagem venosa propostas por Gerrard ${ }^{8}$ e Salvi ${ }^{9}$ podem ser a veia braquial comum, isso devido à semelhança de topografia e de envolvimento de tributárias, alterando-se apenas a nomenclatura dessas veias, podemos concordar com esses autores quanto às estruturas em que a VBC, ou já classificada veia axilar acessória, desembocará.

Concordamos também com Hollinshead ${ }^{10}$ ao afirmar que a VBC pode desembocar na veia axilar, sendo esse o principal destino da VBC.

Em nosso estudo, a maioria das VBC (72\%) desembocou no segmento mais alto da veia axilar (peitoral e suprapeitoral juntas), correspondendo à proposta de Gusmão e Prates ${ }^{11}$, podendo, assim, ser identificada como veia axilar acessória.

Ainda com relação a Gusmão e Prates ${ }^{11}$, concordamos que a veia axilar acessória pode ser formada a partir da VBC, mas não podemos comparar a existência de veia axilar acessória formada por outras veias do braço. Para nós, o estudo se deu apenas com a VBC.

Discordamos da opinião de Santos e Gusmão ${ }^{12}$ quanto ao que chamam de veia axilar acessória; para esses autores, toda veia braquial comum que se estende além do limite superior do braço é uma veia axilar acessória.

Do ponto de vista prático, a VBC esteve presente em $73 \%$ dos cadáveres estudados, o que pode nos garantir frequência elevada de uma veia responsável por importante fração da drenagem venosa do membro superior.

Dessa forma, pode-se efetuar a ligadura da veia axilar em situações críticas sem maiores danos à drenagem venosa do membro superior como citado por Demetriades ${ }^{4}$. Nos casos de trombose venosa profunda em veia axilar ou braquiais $^{6}$, poupando-se a VBC, pode-se pensar em manutenção da drenagem venosa do membro superior por essa veia.

A veia braquial comum está frequentemente presente e, na maior parte das vezes, desemboca na veia axilar.

\section{Referências}

1. Pitta GBB, Santos CAS. Trauma de vasos axilares. In: Murilo R (Ed.). Trauma vascular. Rio de Janeiro: Revinter; 2006. p. 301-7.
2. Sise MJ, Shackford SR. Extremity vascular trauma. In: Rich NM, Mattox KL, Hirshberg A. Vascular Trauma. Elsevier Saunders; 2004. p. 353-89.

3. Bastounis E, Pikoulis E, Leppaniemi AK, Michail P, Alexiou D. Revascularization of the limbs using vein grafts after vascular injuries. Injury. 1998;29:105-8.

4. Demetriades D, Chahwan S, Gomez H, Peng R, Velmahos G, Murray J, et al. Penetrating injuries to the subclavian and axillary vessels. J Am Coll Surg. 1999;188:290-5.

5. Kumar $V$. Endovascular treatment of penetrating injury of axillary vein with Viabahn endoprosthesis. J Vasc Surg. 2004;40: 1243-4.

6. Hingonani A, Ascher E, Marks N, Schutzer RW, Mutyala $M$, Yorkovich $W$, et al. Morbidity and mortality associated with brachial vein thrombosis. Ann Vasc Surg. 2006; 20: 297-300.

7. Prandoni P, Bernardi E, Marchiori A, Lensing AWA, Prins $M H$, Villalta $S$, et al. The long term clinical course of acute deep vein thrombosis of the arm: prospective cohort study. BMJ. 2004;329: 484-5.

8. Gerard G. Manuel d'anatomie humaine. Paris: G. Steinheil. 1912. p. 561-2.

9. Salvi G. Angiologia: capillari, vene, sistema linfatico, milza. In: Bertelli D, Fusari R, Romiti G, Sala L, Salvi G, Sterzi G, et al. Tratatto di anatomia umana. 2. ed. Milano: Casa editrice dottor Francisco Vallardi. 1932. p. 77-9. v. 3.

10. Hollinshead WH, Rose C. Textbook of anatomy. 4. ed. Philadelphia: Harper e Row. 1985. p. 161.

11. Gusmão LCB, Prates JC. Anatomical study of the accessory axillary vein. Surg Radiol Anat. 1992;14:131-6.

12. Santos CAS, Gusmão LCB. Estudo anatômico sobre a veia basílica no braço de cadáveres humanos. Revista do Hospital Universitário da UFAL. 1997;1:32-40.

13. Santos CAS, Figueiredo LFP, Gusmão LCB, Pitta GBB, Miranda Junior F. Válvulas da veia braquial comum: estudo anatômico. J Vasc Bras. 2007;6:35-41.

Correspondência: Carlos Adriano Silva dos Santos Núcleo de Ensino Permanente do SAMU/Maceió Rua Oldemburgo da Silva Paranhos, 800 - Farol CEP: 57055-320 - Maceió (AL) E-mail: carlos_adriano@hotmail.com

Contribuição dos autores: Concepção e desenho do estudo: CASS, LFPF Análise e interpretação dos dados: CASS, LFPF Coleta de dados: CASS, ECFS Redação do artigo: CASS, LFPF, LCBG Revisão crítica do texto: CASS, LFPF, LCBG Aprovação final do artigo: CASS, LFPF, LCBG, ECFS Análise estatística: CASS, LFPF Responsabilidade geral pelo estudo: CASS 